\title{
Correlation between Serum Lipid Profile with Anthropometric and Clinical Variables in Patients with Type 2 Diabetes Mellitus
}

\author{
Belete Biadgo $^{1 *}$, Solomon Mekonnen Abebe ${ }^{2}$, Habtamu Wondifraw Baynes ${ }^{1}$, \\ Mohammed Yesuf ${ }^{3}$, Aynadis Alemu ${ }^{1}$, Molla Abebe ${ }^{1}$
}

OPEN ACCESS

Citation: Belete Biadgo, Solomon Mekonnen Abebe,Habtamu Wondufraw Baynes, Mohammed Yesuf,Aynadis Alemu.Correlation between Serum Lipid Profile with Anthropometric and Clinical Variables in Patients with Type 2 Diabetes Mellitus at University of Gondar Hospital, Northwest Ethiopia. Ethiop J Health Sci 2017;27(3):215. doi: http://dx.doi.org/10.4314/ejhs.v27i3.3

Received: August 9, 2016

Accepted December 10, 2016

Published: May 1, 2017

Copyright: () 2017 Belete B, et al. This is an open access article distributed under the terms of the Creative Commons Attribution License, which permits unrestricted use, distribution, and reproduction in any medium, provided the original author and source are credited.

Funding: University of Gondar Competing Interests: The authors declare that this manuscript was approved by all authors in its form and that no competing interest exists. Affiliation and Correspondence:

${ }^{1}$ Department of Clinical Chemistry,

School of Biomedical and Laboratory

Sciences, University of Gondar,

Ethiopia

${ }^{2}$ Institute of Public Health, College of

Medicine and Health Sciences,

University of Gondar, Ethiopia

${ }^{3}$ Department of Paraclinical Studies,

Faculty of Veternary Medicine,

University of Gondar, Ethiopia

*Email: beletebiadigo@yahoo.com

\begin{abstract}
BACKGROUND: The problem of dyslipidemia is high in patients with diabetes mellitus. There is ample evidence that abnormalities in lipid metabolism are important risk factors for increased incidence of diabetes associated complications. The most important risk indicators for these complications are lipid profile abnormalities. Therefore, the aim of this study was to assess the correlation between serum lipid profile with anthropometric and clinical variables among type 2 diabetes mellitus patients.

METHODS: A comparative cross sectional study was conducted at University of Gondar Hospital from February to April in 2015. A total of 296 participants (148 case and 148 healthy controls) were selected using systematic random sampling technique. Socio- demographic characteristics and clinical data were collected using pretested structured questionnaire incorporating the WHO Stepwise approach. Fasting venous blood sample was collected for blood sugar; lipid profile investigations and the blood levels were determined by Bio Systems A25 Chemistry Analyzer (Costa Brava, Spain). Independent sample t-test and Man Whitney $U$ test were used to compare means. P-value $<0.05$ was considered statistically significant.

RESULTS: Overall, T2DM patients had significantly higher total cholesterol ([205.4 $\pm 50.9 v s 184.9 \pm 44.1] \mathrm{mg} / \mathrm{dl}), \quad$ low density lipoprotein $([113.1 \pm 43.2 v s 100.1 \pm 36.4] \mathrm{mg} / \mathrm{dl})$ and triacylglycerol ([189.22 $100.9 \mathrm{vs} 115.13 \pm 59.2] \mathrm{mg} / \mathrm{dl})$, and significant decline of high density lipoprotein cholesterol $([56.5 \pm 20.4 v s 62.1 \pm 13] \mathrm{mg} / \mathrm{dl})$ as compared to healthy controls, respectively. Triacylglycerolemia was significantly associated with the risk of cardiovascular disease (AOR: 1.015; 95\%CI: 1.010-1.021). Evident correlation was observed between anthropometric and clinical variables with lipid profile.

CONCLUSIONS: Higher serum levels of fasting blood sugar, total cholesterol, low density lipoprotein cholesterol, and triacylglycerol and lower levels of high density lipoprotein cholesterol are found in type 2 diabetes mellitus patients. Thus, DM patients are more prone to dyslipidemia which is an
\end{abstract}


important risk factor for atherosclerosis and coronary heart disease.

KEYWORDS: Type 2 diabetes mellitus, lipid profile, Ethiopia

\section{INTRODUCTION}

Diabetes mellitus (DM) is a group of metabolic disorder of multiple etiologies. It is characterized by chronic hyperglycemia with disturbances of carbohydrate, protein and lipid metabolism resulting from defects in insulin secretion, action, or both (1).

Diabetes Mellitus can be grouped into different clinical classes. $5-10 \%$ of diabetes cases is type $1 \mathrm{DM}$. It is mostly diagnosed during childhood ages and caused by $\beta$-cell damage resulting in diminished ability of the pancreas to produce insulin. The second class is type 2 diabetes mellitus (T2DM). It constitutes over $80-90 \%$ of all DM cases and is mostly diagnosed after the age of 40 . However, younger cases are being reported recently. Insulin is usually produced but not properly utilized due to insulin resistance (IR) in the target cells and gestational DM which is defined as first recognition of DM during pregnancy $(2,3)$.

The major chronic complications of DM are microvascular and macrovascular (4). The overall temporal burden of hyperglycemia is responsible for the complications and adverse outcomes (5). These complications are common in sub-Saharan Africa due to various reasons like lack of community awareness, poor laboratory and clinical diagnosis. As a result of the above reasons, people with DM regularly visit the health facilities because of complication rather than for routine medical checkup (6). It requires continuing medical care and ongoing patient self-management education and support to prevent and reduce complications (7). Multiple risk factors are responsible for the development of T2DM such as behavioral and environmental risk factors (8). Concordance among monozygotic twins is close to $100 \%$, and $25 \%$ of those with the disease have a family history of DM (9). The environmental factors in the pathogenesis of T2DM are obesity, physical inactivity, increase in alcohol intake, over eating and smoking (10).
Diabetes Mellitus currently affects about $5 \%$ of the world's population, and its prevalence is rapidly increasing particularly in elderly people $(11,12)$. It is evolving as one of the most important chronic health problems in Ethiopia (13). The estimated prevalence of DM in adult population of Ethiopia is $1.9 \%$ (13). WHO estimated the number of diabetic cases in Ethiopia as 800,000 by the year 2000 , and the number is expected to increase to 1.8 million by 2030 (15). A community based study in Gondar, Northwest Ethiopia, showed that the prevalence of DM among adults aged 35 years and above was $5.1 \%$ for urban and $2.1 \%$ for rural dwellers (16).

Lipid abnormalities are prevalent in DM patients because of IR which affects key enzymes and pathways in lipid metabolism: Apo protein production, regulation of lipoprotein lipase, action of cholesterol ester transfer proteins and hepatic and peripheral actions of insulin (17). Hyperglycemia and the high level of IR associated with T2DM has multiple effects on fat metabolism which results in the production of atherogenic dyslipidemia characterized by lipoprotein abnormalities: elevated very low density lipoprotein cholesterol (VLDL) elevated low density lipoprotein cholesterol (LDL-c), elevated triacylglycerol (TAG) and decreased high density lipoprotein cholesterol (HDL-c) which are measured for cardiovascular risk prediction (18-22).

The duration of diabetes, degree of hyperglycemia, hypertension, dyslipidemia and smoking are the strongest risk factors for chronic complications of DM that leads to biochemical aberrations (23). Appropriate management targeting glycemic, hypertension and lipid control is important for decreasing morbidity and mortality, and improving long term quality of life for patients diagnosed with T2DM. Lifestyle changes such as nutrition therapy, weight loss, regular physical exercise, appropriate education and self-management strategies are vital to improve outcomes $(24,25)$. Therefore, the aim of this study was to assess the correlation between lipid profile with anthropometric and clinical variables in patients with T2DM at University of Gondar Hospital, Northwest Ethiopia. 


\section{METHODS AND MATERIALS}

Study design, period and area: A comparative cross-sectional study was conducted from February to April, 2015 at Gondar University Hospital Chronic Illness Clinic, Northwest Ethiopia. Gondar Town is $738 \mathrm{~km}$ far from Addis Ababa, the capital city of Ethiopia. The town has around nine government health centers and one university hospital. University of Gondar Hospital provides health service for more than 5 million inhabitants in northwest Ethiopia. It plays an important role in teaching, research and community service. The hospital gives service for more than 8000 diabetic patients.

Sample size and study subjects: The sample size was calculated based on two population mean formula using Open Epi Info version 2, open source calculator by considering $95 \%$ confidence interval (2-sided) and $80 \%$ power. Ratio of sample size (T2DM group/control group) is 1 . We used mean \pm SD of $4.41 \pm 1.39$ and $3.98 \pm 1.24$ for cases and controls, respectively (26). Therefore, the total sample size was 296 (equal number of cases and controls). All T2DM patients attended to the institution were included using systematic random sampling technique. All T2DM patients attending at University of Gondar Hospital were included in the study.In addition, age and sex matched apparently healthy individuals were employed as a control group. On the other hand, patients who already had any chronic diseases, pregnant women and patients on statins for abnormal lipid treatment and on insulin therapy, smokers, alcoholics and those who were on anticoagulant drugs were excluded from the study.

Data collection and laboratory methods: Patients were asked to fill the written consent form after pre-test was conducted in $5 \%$ of the study participants. Data on socio-demographic characteristics were collected by trained nurses from University of Gondar Hospital Chronic Illness Clinic by using semi-structured questionnaire. Anthropometric variables including body mass index (BMI) and waist circumference (WC) were measured. BMI was calculated as weight in kilogram divided by height in centimeter square. WC was taken midway between the lowest rib and the iliac crest. Cut off values for anthropometric and lipid profile values were based on the National Cholesterol Education Program-Adult Treatment Panel III (NCEP-ATP III) guidelines. Abnormal lipid profile was defined as $\mathrm{TC} \geq 200 \mathrm{mg} / \mathrm{dl}$, HDL-c < 40mg/dl, LDL-c $\geq 130 \mathrm{mg} / \mathrm{dl}$, and TAG $\geq 150 \mathrm{mg} / \mathrm{dl}$ (19). An elevation of any one of the lipid parameters to a level above these limits was considered as dyslipidemia. Blood pressure was taken by qualified personnel using an analogue sphygmomanometer and stethoscope. Duplicate measurements were taken from the upper arm with the hand at the heart level after the patient had been sitting for more than 5 minutes and the averaged value was recorded. Five milliliter blood sample was collected from the patients by a laboratory technologist for lipid profile investigation after 10-12 hour fasting. Glucose and lipid profile levels were measured by glucose oxidase and direct enzymatic methods, respectively using Bio systems A25 (Costa Brava, Spain) automated clinical chemistry analyzer according to manufacturer's instructions.

Data management and quality control: The questionnaire was pre-tested for accuracy and consistency prior to actual data collection and a half day training was given to data collectors. Furthermore, the principal investigator gave feedback and corrections on daily basis to the data collectors. The completion, accuracy, and clarity of the collected data were checked carefully on a regularly basis. Normal and pathological control samples were run daily in order to check the optimal reactivity of the reagent and functionality of the analyzer. The results obtained from control samples should be within mean $\pm 2 \mathrm{SD}$ of the given Levy Jenning chart. Therefore, to maintain the quality of the result, pre-analytical, analytical and postanalytical precautions of quality, depending on the stated SOP, were considered. Appropriate remedies were taken in cases of unacceptable quality control results. Finally, the sample was processed within 1 hour of specimen collection in the clinical chemistry laboratory and processed based on the manufacturer's manual.

Data analysis and interpretation: Data were entered and analyzed using SPSS version 20 
(IBM, USA). The data were tested for normality with the help of histograms, comparison of means and medians, skewness and the Kolmogorov-Smirnov test. Data were reported as mean and standard deviation for continuous variables, percentages for categorical variables and interquartile range for non-normally distributed data. Variables were compared using independent sample t-test for normally distributed data and Mann-Whitney $U$ test for non-normally distributed data. Pearson's and spearman's rank correlations were used to test the relationship of lipid profile with clinical and anthropometric variables. P-value $<0.05$ was considered statistically significant.

Ethical consideration: Ethical clearance was taken from Research and Ethical Committee of the School of Biomedical and Laboratory Sciences, University of Gondar, and permission was secured from both the Medical Director and the head of the Chronic Illness Clinic of the Hospital. In addition, written informed consent was obtained from the study participants before data collection.

\section{RESULT}

Socio-demographic characteristics of the study participants: A total of 296 (148 T2DM and 148 controls) study participants were participated in this study. Of the total T2DM patients, 59(39.9\%) and 89(60.1\%) were males, females respectively. Similarly, of the 148 healthy controls $89(60.1 \%)$ were females. The overall mean age was $49.09 \pm 8.1$ and $47.8 \pm 6.7$ years for T2DM and controls respectively. The residence of the majority, 278 (93.9\%), of the participants was urban. Summary of the other demographic variables are presented in (Table $1)$.

Table 1: Socio-demographic characteristics of study participants at University of Gondar Hospital, Northwest Ethiopia, 2015 ( $\mathrm{N}=296)$.

\begin{tabular}{llll}
\hline Variables & T2DM \% $(\mathbf{N}=\mathbf{1 4 8})$ & Controls \% $(\mathbf{N}=\mathbf{1 4 8})$ & P-value \\
\hline Age in year & & & 0.071 \\
$\mathbf{2 5 - 3 4}$ & $5(3.4)$ & $4(2.7)$ & \\
$\mathbf{3 5 - 4 4}$ & $32(21.6)$ & $36(24.3)$ & \\
$\mathbf{4 5 - 5 4}$ & $72(48.7)$ & $83(56.8)$ & \\
$\mathbf{5 5 - 6 4}$ & $36(24.3)$ & $24(16.2)$ & \\
$\mathbf{6 5 +}$ & $3(2.0)$ & $1(0.7)$ & 1.00 \\
Sex & & & \\
Male & $59(39.9)$ & $59(39.9)$ & 0.052 \\
Female & $89(60.1)$ & $89(60.1)$ & \\
Residence & & & \\
Urban & $135(91.2)$ & $143(96.6)$ & 0.745 \\
Rural & $13(8.8)$ & $5(3.4)$ & \\
Religious & & $139(93.9 \%)$ & \\
Orthodox & $135(91.2)$ & $5(3.4)$ & 0.135 \\
Muslim & $11(7.4)$ & $4(2.7)$ & \\
Protestant & $2(1.4)$ & & \\
Marital status & & $16(10.8)$ & \\
Single & $8(5.4)$ & $108(73.0)$ & \\
Married & $106(71.6)$ & $12(8.1)$ & \\
Divorced & $12(8.1)$ & $10(6.8)$ & \\
Widowed & $19(12.8)$ & $2(1.4)$ & \\
Separated & $3(2.0)$ & & \\
\hline
\end{tabular}

DOI: http://dx.doi.org/10.4314/ejhs.v27i3.3 
Clinical features and anthropometric measurements of the study participants: The result showed that the mean levels of BMI, WHR, systolic BP and diastolic BP were significantly higher $(P<0.05)$ in T2DM compared to controls. The median duration of illness since diagnosis was 3 years for T2DM patients. From the total study participants, $72(48.6 \%)$ and $35(23.6 \%)$ were overweight cases and controls, respectively. The mean WC showed statistically significant difference between the two groups $(83.02 \pm 12.6$ and $78.67 \pm 10.1$ ), $\mathrm{p}<0.001$ respectively (Table 2 ).

Table 2: Clinical features and anthropometric measurements of the study participants at University of Gondar Hospital, Northwest Ethiopia, 2015 (n=296).

\begin{tabular}{|c|c|c|c|}
\hline Variables & T2DM\% (N=148) & Controls \% $(\mathrm{N}=148)$ & P-Value \\
\hline BMI $\left(\mathrm{Kg} / \mathrm{m}^{2}\right)$ & & & 0.000 \\
\hline Underweight & $2(1.4)$ & $7(4.7)$ & \\
\hline Healthy weight & $62(41.9)$ & $101(68.2)$ & \\
\hline Over weight & $72(48.6)$ & $35(23.6)$ & \\
\hline Obese & $12(8.1)$ & $5(3.4)$ & \\
\hline $\mathrm{WC}(\mathrm{cm})$ & & & 0.001 \\
\hline Low risk & $85(57.4)$ & $109(73.7)$ & \\
\hline Increased risk & $25(17)$ & $14(9.5)$ & \\
\hline High risk & $38(25.7)$ & $25(17)$ & \\
\hline WHR $^{\odot}$ & $0.88 \pm 0.88$ & $0.84 \pm 0.66$ & 0.000 \\
\hline \multicolumn{4}{|l|}{ Blood pressure (mmHg) } \\
\hline Systolic BP & $130(120-140)^{*}$ & $110(110-120)^{*}$ & 0.000 \\
\hline Diastolic BP & $80(70-88)^{*}$ & $70(70-80)^{*}$ & 0.000 \\
\hline \multicolumn{4}{|l|}{ Duration since diagnosis (in year) } \\
\hline$<5$ & $107(72.3)$ & - & \\
\hline$\geq 5$ & $41(27.7)$ & - & \\
\hline Median duration of DM in year & $3(1-6) *$ & & \\
\hline
\end{tabular}

Note: *Presented as median and interquartile range, () Presented as mean \pm standard deviation BMI: Body Mass Index, WHR: Waist to Hip Ratio, FBS: Fasting Blood Sugar, BP: Blood Pressure, WC: Waist Circumference

Comparison of lipid profile and Fasting blood sugar level of the study participants: Significant increase in TC, LDL-c, TAG and FBS concentrations were observed in diabetic patients $(205.4 \pm 50.9$ vs $184.9 \pm 44.1,113.1 \pm 43.2$ vs $100.1 \pm 36.4,189.22 \pm 100.9$ vs $115.13 \pm 59.2$ and $163.7 \pm 1.33$ vs $81.3 \pm 1.08 \quad(\mathrm{p}<0.05)$ as compared to the control group respectively. On the contrary, there was a significant decline in mean concentration of HDL-c in T2DM compared to controls $(56.5 \pm 20.4$ vs $62.1 \pm 13)$ $\mathrm{p}=0.007$ respectively (Table 3$)$. 
Table 3: Comparison of lipid profiles and Fasting blood sugar concentration of the study participants using t-test at University of Gondar Hospital, Northwest Ethiopia, 2015 (n=296).

\begin{tabular}{llll}
\hline Variables & Mean \pm SD T2DM & Mean \pm SD Controls & P- value \\
\hline Lipid profiles (mg/dl) & & & \\
Total cholesterol & $205.4 \pm 50.9$ & $184.9 \pm 44.1$ & 0.000 \\
Triacyl glycerol & $189.22 \pm 100.9$ & $115.13 \pm 59.2$ & 0.000 \\
High density lipoprotein & $56.5 \pm 20.4$ & $62.1 \pm 13.5$ & 0.007 \\
Low density lipoprotein & $113.1 \pm 43.2$ & $100.1 \pm 36.4$ & 0.009 \\
Fasting blood sugar (mg/dl) & $163.7 \pm 1.33$ & $81.3 \pm 1.08$ & 0.000 \\
\hline
\end{tabular}

\section{Note: SD: Standard deviation, T2DM: Type 2 Diabetes Mellitus}

The most commonly observed lipid followed by hypercholesterolemia 51(34.5\%), abnormalities in the study was TAG 94(63.5\%) hypertriacylglycerolemia $40(27 \%)$ and low followed by raised LDL92 (62.2\%), hypercholesterolemia $80(54.1 \%)$ and low HDL cholesterolemia $24(16.2 \%)$ for T2DM patients. Raised LDL cholesterolemia 76(51.4\%) HDL cholesterolemia 2(1.4\%) were reported in controls. The proportions of lipid profile disorders were higher in DM patients compared to apparently healthy controls (Figure 1).

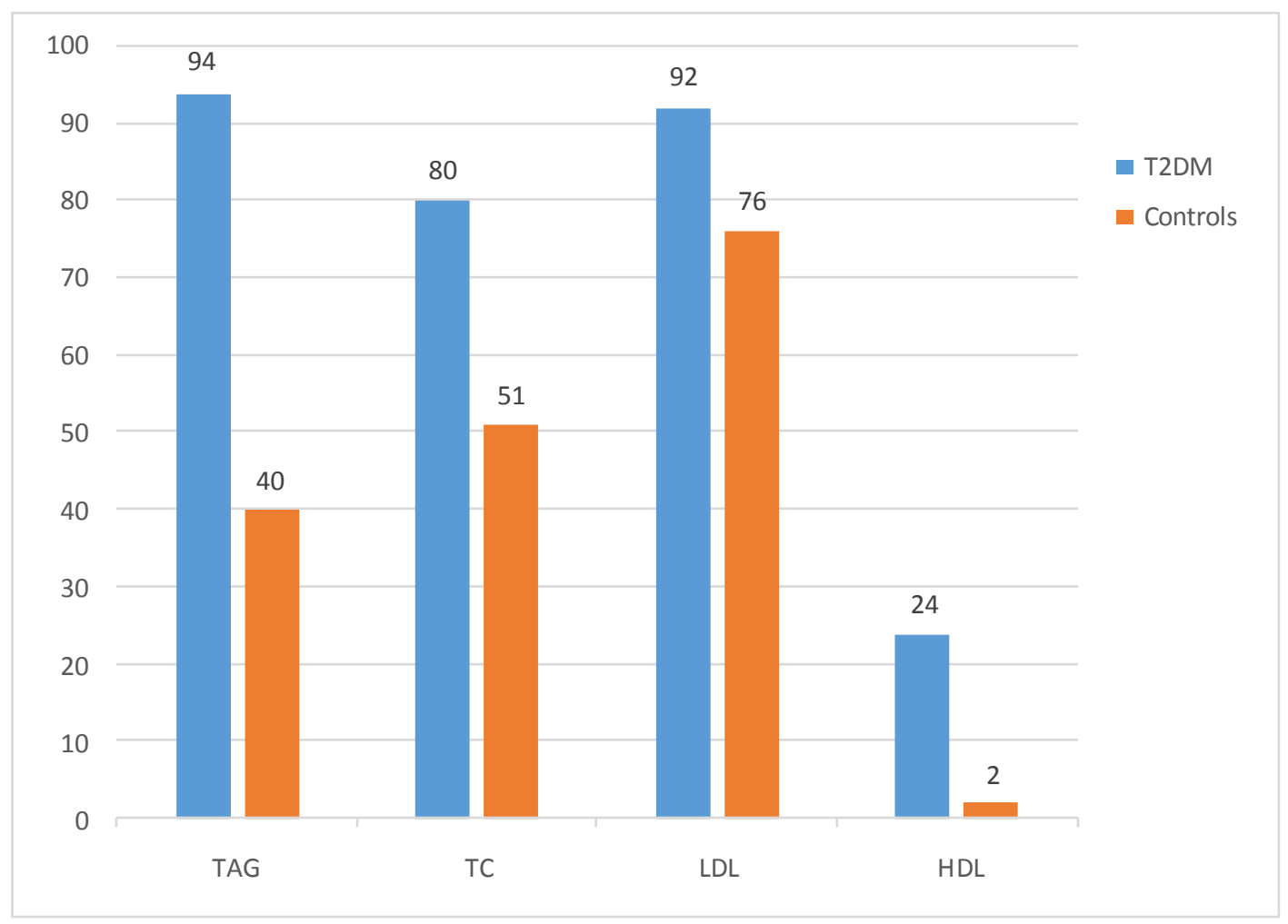

Figure 1: Proportions of diabetes mellitus patients and controls in relation to lipid profile concentrations at University of Gondar Hospital, Northwest Ethiopia, 2015 
The association between CVD risk of lipid parameters effects on type 2 diabetic and non-diabetic participants: The serum lipid profile associated risks were evaluated by using univariate and multivariate logistic regression analysis and summarized in (Table 4). Bivariate logistic regression analysis revealed that the higher lipid values of TC, TAG, HDL cholesterol and LDL cholesterol were significantly $(\mathrm{p}<0.001)$ associated with the risk of developing CVD among T2DM and control subjects. Further, adjusted multivariate logistic regression analysis confirmed that $\mathrm{TAG}$ $(\mathrm{OR}=1.015, \quad 95 \% \quad \mathrm{CI}=1.010-1.021, \quad \mathrm{p}=0.000)$ were the significant and independent risk factors for CVD among T2DM patients (Table 4).

Table 4: The association between cardiovascular disease risk and serum lipid concentrations of T2DM and non-diabetic subjects at University of Gondar Hospital, Northwest Ethiopia, 2015

\begin{tabular}{lllll}
\hline Variable & Unadjusted OR (95\% CI) & P-Value & Adjusted OR (95\% CI) & P-Value \\
\hline Total cholesterol & $1.009(1.004-1.014)$ & $0.000^{*}$ & $1.003(0.989-1.017)$ & 0.690 \\
$\begin{array}{l}\text { Triacylglycerol } \\
\text { High density }\end{array}$ & $1.016(1.012-1.021)$ & $0.000^{*}$ & $1.015(1.010-1.021)$ & $0.000^{*}$ \\
$\begin{array}{l}\text { lipoprotein } \\
\text { Low density }\end{array}$ & $0.982(0.969-0.995)$ & $0.008 *$ & $0.992(0.972-1.012)$ & 0.415 \\
lipoprotein & $1.008(1.002-1.014)$ & $0.010^{*}$ & $1.002(0.986-1.018)$ & 0.817 \\
\hline
\end{tabular}

Note: OR: Odds Ratio, CI: Confidence Interval, *: Statistically significant association

Correlations between lipid profiles with FBS, Duration of DM and Anthropometric measurements of study participants: There was non-significant negative correlation between BMI and HDL, where as a statistically significant positive correlation existed in other lipid profiles in both diabetic and control groups. Waist to hip ratio (WHR) achieved a statistical significant positive correlation with Total cholesterol $(\mathrm{p}=0.000)$, Low density lipoprotein $(\mathrm{p}=0.000)$ and Triacylglycerol $(\mathrm{p}=0.015)$ in T2DM (Table 5).

Table 5: Pearson's correlations(r) between lipid profiles with BMI and WHR among T2DM and healthy controls at University of Gondar Hospital, Northwest, Ethiopia, 2015, (n=296).

\begin{tabular}{lllll}
\hline \multicolumn{2}{c}{ Type 2 DM group } & \multicolumn{2}{c}{ Control group } \\
\hline Variables & BMI & WHR & BMI & WHR \\
& $\mathrm{r}(\mathrm{p})$ & $\mathrm{r}(\mathrm{p})$ & $\mathrm{r}(\mathrm{p})$ & $\mathrm{r}(\mathrm{p})$ \\
\hline Low density lipoprotein & $0.293^{* *}(0.000)$ & $0.283^{* *}(0.000)$ & $0.364^{* *}(0.000)$ & $0.349^{* *}(0.000)$ \\
High density lipoprotein & $-0.053(0.519)$ & $-0.025(0.766)$ & $-0.172(0.036)$ & $-0.065(0.431)$ \\
Total cholesterol & $0.167^{*}(0.042)$ & $0.349^{* *}(0.000)$ & $0.231^{* *}(0.005)$ & $0.396^{* *}(0.000)$ \\
Triacylglycerol & $0.335^{* *}(0.000)$ & $0.200^{*}(0.015)$ & $0.499^{* *}(0.000)$ & $0.117(0.156)$ \\
\hline
\end{tabular}

Note: BMI: Body Mass Index, WHR: Waist to Hip Ratio, ** and *: Correlation is significant at 0.01 level and 0.05 level (2-tailed) respectively)

The correlation between systolic BP among various lipid profiles showed statistically significant correlation inT2DM and control groups for TAG. However, diastolic BP was not found to have statistically significant correlation with any of the lipid profiles (Table 6). In the T2DM groups, FBS showed a significant positive correlation with TAG and LDL. There was no significant correlation observed among any of lipid profiles in the control group. Duration of T2DM also significantly correlated with Low density lipoprotein and total cholesterol (Table 6). 
Table 6: Correlations between lipid profiles with Systolic, diastolic blood pressure, FBS and duration of DM among T2DM and healthy controls at University of Gondar Hospital, Northwest, Ethiopia, 2015, $(n=296)$.

Note: SBP: Systolic Blood Pressure, DBP: Diastolic Blood Pressure, FBS: Fasting Blood Sugar, T2DM: Type 2

\begin{tabular}{lllll}
\hline & \multicolumn{2}{c}{ Type 2 DM group } & \multicolumn{2}{c}{ Control group } \\
\hline Variables & $\begin{array}{l}\text { SBP } \\
\text { rho(p) }\end{array}$ & $\begin{array}{l}\text { DBP } \\
\text { rho(p) }\end{array}$ & $\begin{array}{l}\text { SBP } \\
\text { rho(p) }\end{array}$ & $\begin{array}{c}\text { DBP } \\
\text { rho(p) }\end{array}$ \\
\hline Low density lipoprotein & $0.071(0.391)$ & $0.039(0.634)$ & $0.078(0.348)$ & $0.066(0.429)$ \\
High density lipoprotein & $-0.020(0.813)$ & $-0.008(0.927)$ & $-0.008(0.927)$ & $-0.060(0.471)$ \\
Total cholesterol & $0.079(0.342)$ & $0.039(0.634)$ & $0.024(0.772)$ & $0.074(0.369)$ \\
Triacylglycerol & $0.723^{*}(0.001)$ & $0.059(0.473)$ & $0.240 *(0.003)$ & $0.153(0.064)$ \\
\hline Variables & FBS & Duration of T2DM & FBS & \\
& r(p) & r(p) & r(p) & \\
\hline Low density lipoprotein & $0.173^{*}(0.035)$ & $0.303^{* *}(0.000)$ & $0.076(0.359)$ & \\
High density lipoprotein & $-0.101(0.220)$ & $-0.010(0.900)$ & $-0.059(0.477)$ & \\
Total cholesterol & $0.096(0.247)$ & $0.408^{* *}(0.000)$ & $0.089(0.281)$ & \\
Triacylglycerol & $0.225^{* *}(0.006)$ & $0.115(0.166)$ & $0.057(0.495)$ &
\end{tabular}

Diabetes Mellitus, ** and *: Correlation is significant at 0.01 level and 0.05 level (2-tailed) respectively), $\boldsymbol{r}$ : Pearson's correlation coefficient, rho: Spearman's rank correlation coefficient.

\section{DISCUSSION}

This study showed a significant increase in TC, LDL cholesterol and TAG in diabetic patients compared to the control group. In addition, there was a significant decline in mean concentration of HDL cholesterol in T2DM patients compared to controls. These phenomena indicate the occurrence of risk factors for CVD which was documented by several studies in agreement to this study (27-33). Hypertriacylglycerolemia $(63.5 \%)$ was the major lipid parameter disorder in our study. This finding is in concord with previous studies in Hyderabad (60\%) and Sudan (48.8\%) $(28,31)$. This is due to the fact that increased hepatic secretion of VLDL and delayed clearance of TAG rich lipoproteins increased levels of substrates for TAG production, enhanced mobilization of free fatty acid (FFA) from adipose tissue and increased in the blood of DM patients as observed in the present study (34). Elevated TAG levels are a common dyslipidemic feature accompanying T2DM and prediabetes states (35).
A fasting TAG level of greater than $150 \mathrm{mg} / \mathrm{dl}$ is one of the accepted criteria for defining individuals at high risk for CVD and T2DM (36).

Our study showed raised TAG, LDL and low HDL cholesterol levels in DM patients. This result is similar to Asian Pacific Cohort Studies Collaboration (37). According to the CDC, 97\% of adults with diabetes have one or more lipid abnormalities (38). On contrary to previous studies, TC and LDL cholesterol were raised in our finding $(37,29)$ in T2DM patients. Another study showed that there is no statistically significant difference in serum TC and LDL cholesterol concentration in diabetic patients compared to control groups which is inconsistent to our study $(29,32)$. In contrast to our result, (16.2\%), HDL cholesterol dyslipidemia in T2DM is higher in previous studies in Hyderabad (71\%), Nepal (20\%), Sudan (93\%) and Ghana (26\%) $(27,28,30,31)$. The variation is due to differences in genetics, life style difference and management of diabetes in the particular group being studied.

DOI: http://dx.doi.org/10.4314/ejhs.v27i3.3 
Previous study reported that lipid compositions of various tissues altered in diabetes (39). Hence, it is postulated that circulatory lipids play a vital role in progression of T2DM, not only by way of lipid abnormalities but also by modifying the composition, structure and firmness of cellular membranes (39). Several studies reported high levels of TAG, TC and LDL-c among diabetic patients, a finding observed in the present study too. However, another study in Nigeria reported a different finding (33) which is higher TAG in controls. The main cause for lipid abnormalities in T2DM patients is impaired secretion of insulin that affects the liver apolipoprotein production and regulates the enzymatic activity of lipoprotein lipase (LpL) and cholesterol ester transport protein (CETP). Moreover, its deficiency reduces the activity of hepatic lipase; therefore, several steps involved in the production of biologically active LpL might be altered in T2DM compared to controls.

Generally, T2DM is associated with low HDL cholesterol levels, which is an independent cardiovascular risk factor. The low concentrations reflect a dysregulation in HDL metabolism. It is determined by the concerted action of different proteins, including CETP, lecithin cholesterol acyltransferase, LpL, phospholipid transfer protein, hepatic lipase and dysfunctional HDLmediated reverse cholesterol transport are impaired in T2DM patients compared to controls (40). The association between dyslipidemia and risk of CVD is biologically plausible and compatible with what is known about pathophysiology of CVD. The hypothesized mechanism for dyslipidemia and CVD association has been described. Investigators noted that elevated plasma lipid and lipoprotein may induce endothelial dysfunction secondary to oxidative stress (22. Other mechanism is pathologic process of CVD via dysregulation of $\mathrm{LpL}$ resulting in a dyslipidemic lipid profile (19).

In this study, we also tried to correlate lipid profiles with various clinical and anthropometric variables. The variables above the threshold cut off values were found to be predictors of CVD in various populations. In the present study, we observed statistically significant increases in the mean values of BMI and the WC in T2DM participants compared to the controls. Similar findings were observed by other researchers $(41,42)$. In the present study, lipid profile correlated in a comparable manner with BMI and WHR. This finding is consistent with a previous study (43) which showed positive correlation between BMI and WHR with respect to TC, TAG. However, previous study reported that correlation existed only in BMI and VLDL (44).

A recent study, in agreement with this study, demonstrated significant differences in anthropometric variables and lipid profile in T2DM in three different ethnic groups in Malaysia (45). In another recent study in Iranian, it was observed that correlation happened between BMI and TC, LDL cholesterol, TAG in concord to our study (46). Another recent study from North India, agreement to this study, reported positive correlations between WHR, TC, LDL cholesterol in healthy subjects in (47).

Other study in Saudi Arabia and Korean, consistent to this study, reported that there was a positive correlation between BMI with TC, LDL and WHR with TC, LDL respectively, but in contrast to ours, no correlation was seen between BMI and TAG in Saudi Arabia study $(48,49)$. A similar study in Brazil demonstrates an association between the lipid profiles and body adiposity (50).

Interrelation between level of BP and lipid profiles may be through diabetes related to the development of hypertension and dyslipidemia $(51,52)$. In our study, the mean systolic and diastolic BP is significantly higher in T2DM patients compared to healthy controls. This is due to peripheral resistance which is a major factor influencing diastolic BP, influenced by narrowing of blood vessels in T2DM patients that increases diastolic BP (53). In contrast to our study, a prospective analytical study found a significant positive correlations of TC, LDL cholesterol with BP (54). A study on patients diagnosed as hypertension found that TC and LDL cholesterol were associated closely with level of BP (55). Another recent cross sectional survey on 32004 patients showed that co-existence of high BP and abnormal glucose metabolism was common in Chinese population (56). A study demonstrated an association between hypertension and hypertriglycerolemia with poor glycemic control

DOI: http://dx.doi.org/10.4314/ejhs.v27i3.3 
consistent to our study (57). Noteworthy is the fact that different to previous studies, our results did not show any correlation between BP and lipid profile in T2DM patients except TAG.

In our study, FBS had statistically significant correlation with TAG and LDL cholesterol in T2DM. Congruent to our study, a study in India reported higher TC, TAG and LDL cholesterol in patients who have poor glycemic control (58), in Nepal on T2DM reported that significant correlations with TC, LDL cholesterol, and significant negative correlation with HDL cholesterol (59). In contrast to our study, a study on healthy population in India showed, significant correlation of FBS with TAG, LDL and negative correlation to HDL cholesterol. Similar to our study, no correlation of FBS with TC in control groups (60).Duration of DM achieved significant correlation with LDL cholesterol, TC. Studies in India found that chronicity of T2DM cause higher lipid disturbance (57) which is observed in our study. According to United Kingdom prospective study risk score for CVD $(61,62)$, duration of diabetes was associated with higher incidence of dyslipidemia of TC, LDL cholesterol in agreement to our study.

In conclusion, there was statistically significant difference in lipid profile level between diabetic patients and apparently healthy controls. The study also reported that significant increase in FBS, systolic and diastolic BP, BMI, WC in diabetics than apparently healthy controls. Significant correlation was observed in lipid profiles with anthropometric and clinical variables. This is a reflection of poor glycemic control and life style changes. This suggests a necessity in educating T2DM patients about regular follow-up and appropriate glycemic control to prevent and delay the progression of the disease. Periodic medical checkup and appropriate life style change is recommended for apparently healthy controls to improve the quality of interventions not only to achieve weight loss goals but also to control dyslipidemia and reduce cardiovascular risk. Thus multi-center prospective studies with larger cohort may be needed in the future.

\section{ACKNOWLEDGEMENT}

We express our deepest gratitude to all of the study participants. We also would like to acknowledge the University of Gondar Hospital laboratory staff members and Mr. Desalew Wudineh for their cooperation during data collection.

\section{REFERENCES}

1. American Diabetes Association. Diagnosis and Classification of Diabetes Mellitus. Diabetes care.2008; 31 (1):S55-S60.

2. Annette H, Hilde L and Elisabete W. Obesity and diabetes epidemics cancer repercussions. Innovative endocrinology of cancer, edited by LevM. Berstein and Richard J.Santen, 2008; 73.

3. American Diabetes Association. Standards of Medical Care in Diabetes. Diabetes Care .2014; 37(1):S14.

4. Fauci A, Braunwald E, Kasper D, Hauser S, Longo D. Harrison's Principles of Internal Medicine. $17^{\text {th }}$ edition. United States of America. The McGraw Hill Company; 2008.

5. Rizvi A, Sanders MB. Assessment and monitoring of Glycemic control in primary diabetes care: monitoring techniques, record keeping, tests of average glycaemia, and point of care evaluation. $J$ Am Acad Nurse Pract .2006; 18:11-21.

6. Africa diabetes leader ship forum (DLF). Diabetes: the hidden pandemic and its impact on sub-Saharan Africa. Johannesburg 2010.

7. American diabetes association. Standards of medical care in diabetes. Diabetes cares.2013; 36 (1): S11.

8. Rother KI. Diabetes treatment bridging the divide. N Engl J Med.2007; 356(15):1499-1501.

9. Florez JC. Clinical review: the genetics of type 2 diabetes: a realistic appraisal in 2008. J Clin Endocrinol Metab.2008; 93: 4633-4642.

10. Kaku K. Pathophysiology of type $2 \mathrm{DM}$ and its treatment policy. JMAJ .2010; 53(1):41-46.

11. Reinehr T. Type 2 diabetes mellitus in children and adolescents. World J Diabetes. 2013; 4(6): 270281.

12. Laakso M. Heart in diabetes: a microvascular disease. Diabetescare. 2011; 34 (2): S145-S149.

13. Abdulkadir J, Reja A. Management of diabetes mellitus: Coping with limited facilities. Ethiop Med J .2001; 39:349-365. 
14. Alemu S, Dessie A, Seid E, Bard E, Lee PT, Trimple ER, et al. Insulin requiring diabetes in rural Ethiopia, Should we reopen the case for malnutrition related diabetes? Diabetologia. 2009; 52:1842-1845.

15. Naheed G. Knowledge, Attitudes and Practices of Type 2 Diabetics Mellitus. J Ayub MedColl Abbottabad. 2010; 22(3).

16. Abebe SM, Berhane Y, Worku A, Assefa A. Diabetes mellitus in North West Ethiopia: a community based study. BMC public health. 2014; 14(1):97.

17. Frank B, Stampfer J, Steven M. Elevated risk of cardiovascular disease prior to clinical diagnosis of type 2 diabetes. Diabetes Care.2002; 25:1129-34.

18. Shahid SH. Frequency distribution of atherogenic dyslipidemia in Saudi type 2 diabetic patients. Pak J physiol.2006; 2(2):20-23.

19. Grundy SM, Cleeman JI, Merz CN, Brewer HB Jr, Clark LT, Hunning hake DB. Implications of recent clinical trials for the National Cholesterol Education Program Adult Treatment Panel III guidelines. Circulation.2004; 110(2):227-39.

20. Nigam PK. Serum Lipid Profile: Fasting/Nonfasting? IndJ Clin Biochem.2011; 26(1):96 97.

21. Mullugeta Y, Chawla R, Kebede T, Worku Y. Dyslipidemia Associated with Poor Glycemic Control in Type 2 Diabetes Mellitus and the Protective Effect of Metformin Supplementation. Ind J ClinBiochem. 2012; 27(4):363-369.

22. Maitra A. The Endocrine System. In: Kumar V, Abbas AK, Fausto N, Aster JC, editors. Robbins and Cotran Pathologic Basis of Disease. $8^{\text {th }} \mathrm{ed}$. New Delhi: Elsevier; 2010, 1097-164.

23. Marshall SM, Flyvbjerg A: Prevention and early detection of vascular complications of diabetes. BMJ. 2006; 333:475-480.

24. Redmon B, Caccamo D, Flavin P, Michels R, Myers C, O'Connor P, et al. Institute for Clinical Systems Improvement. Diagnosis and Management of Type 2 Diabetes Mellitus in Adults. 2014.

25. Solano MP and Goldberg RB. Lipid Management in Type 2 Diabetes. Clini.Diabetes.2006; 24(1):16.

26. Samuel AA, Pius DO, Adeyemi OB, Adeola TNA , Jimoh AK, Adepeju AA. Liver Enzymes and Lipid Profile among Type 2Diabetic Patients in Osogbo, Nigeria. Greener J.Medical Sciences.2013; 3 (5):174-178.

27. Ramu K, Vinayak E, Shegokar. A study of lipid profile in patients with type 2 diabetes mellitus. Journal of health sciences.2013; 1(1): 23-27.
28. Shaikh MA, Kumar S, Ghouri RA. Type 2 diabetes mellitus and lipid abnormalities. JLUMHS. 2010; 9(03): 145 .

29. Dahal S, Baral BK, Baral S, Shrestha R and Khanal M. Study of fasting serum lipid and lipoproteins profile in type II diabetic patients. Nepal Med Coll J.2013; 15(1): 18-22

30. Tagoe DNA, Amo-Kodieh P. Type 2 diabetes mellitus influences lipid profile of diabetic patients. Annals of Biological Research.2013; 4 (6):88-92.

31. Elnasril H.A. and Ahmed A.M. Patterns of lipid changes among type 2 diabetes patients in Sudan. Easte. Mediter. Health Journal.2008; 14(2): 314324.

32. Sahibzada SJ, AmirRehman, RA, Taj MK, Aziz A, Amjad A. Evaluation of pattern of dyslipidemia in type 2 diabetics in swat. Gomal J. Medic Sciences. 2011; 9 (2):243-247.

33. Oluyomia EOB, Fisayo AM, Bayodea FJ, Kehindea FM and Ademigbuji E. Adedayoa. Lipid profile of a group of Nigerian diabetic patients. Archives of Appli. Sci .Research.2010; 2 (4): 302306.

34. Mooradian AD.Dyslipidemia in type 2 diabetes mellitus. Nat Clin Pract Endocrinol Metab. 2009; $5: 150-9$.

35. Ginsberg HN, Zhang YL, Hernandez-Ono A: Regulation of plasma triglycerides in insulin resistance and diabetes. Arch MedRes.2005; 36:232-240.

36. Kahn R, Buse J, Ferrannini E, Stern M. The metabolic syndrome: time for a critical appraisal. Joint statement from the American Diabetes Association and the European Association for the Study of Diabetes. Diabetologia. 2005; 48:16841699.

37. Kengne PA, Patel A, Barzi F, Jamrozik K, Lam HT, Ueshima T, et al. Cholesterol, diabetes and major cardiovascular diseases in the Asia-Pacific region. Diabetologia.2007; 50:2289-97.

38. Ansorge R. Lowering high triglycerides, cholesterol is very important in diabetes. 2008.

39. Letícia AS, Deoliveira MS, Paula Salles AMF, Das Graças MC. Hemostatic changes in patients with type 2 diabetes mellitus. Rev Bras Hematol Hemoter.2010; 32 (6):482-488.

40. Linthout SV, Spillmann F, Schultheiss HP, Tschöpe C. High-Density Lipoprotein at the Interface of Type 2 Diabetes Mellitus and Cardiovascular Disorders. Current Pharmaceutical Design, 2010, 16(13), 1504-1516.

41. Hardev SS, Shyamal K, Karanjit SS. A study of correlation between lipid profile and waist to hip

DOI: http://dx.doi.org/10.4314/ejhs.v27i3.3 
ratios in patients with diabetes mellitus. Anthropologist.2008; 10:215-8.

42. Srinivas C, Lakshmana NK, Mallikarjuna R, Kishore K, Kiran D, Surya NK. Relationship between anthropometric, metabolic parameters and fasting glucose in rural population of Andhra Pradesh. J Evolution Med Dent Sci.2014; 3:90949.

43. Raja Reddy P, Jayarama N, Mahesh V. Anthropometric Correlation of Lipid Profile in Healthy People in Rural Kolar. Interdiscip. Resea. Journal.2013; 3(4):2249-9598.

44. Himabindu Y, Sriharibabu M, Alekhya K, Saisumanth K, Lakshmanrao N, Komali1 K. Correlations between anthropometry and lipid profile in type 2 diabetics. Indian J Endocrinol Metab. 2013; 17(4): 727-729.

45. Blebil AQ, Hassan Y, Dujaili JA. Differences in demographics, lipid profile and other clinical characteristics among type 2 diabetic patients in the state of Penang, Malaysia according to gender and races. Asian J Pharm Clin Res. 2011; 4 (2):130-3.

46. Ghorbanian B. Relation between anthropometric indicators and serum lipid profiles as cardiovascular risk factors personals of Iranian Azarbayjan University of ShahidMadani. Euro. J. Sports Exer. Sci. 2012; 1 (3):52-58.

47. Sandhu HS, Koley S, Sandhu KS. A study of correlation between lipid profile and waist to hip ratios in patients with diabetes mellitus. Anthropologist. 2008; 10:215-8.

48. Al-Ajlan AR. Lipid Profile in Relation to Anthropometric Measurements among College Male Students in Riyadh, Saudi Arabia. Int $J$ Biomed Sci .2011; 7 (2): 112-119.

49. Sarah C, Edwin T. Anthropometric measures and lipid CHD risk factors in Korean Immigrants with Type 2 Diabetes. Cardiovasc Nurs. 2011; 26:414.

50. Lisianny CCdN, Ferreira, Humberto JGdaS, Tatiana AL, Wagner LdoP. Relationship between lipid and hematological profiles with adiposity in obese adolescents.Rev Bras Hematol Hemoter. 2013; 35 (3):163-6.

51. Petitti DB, Imperatore G, Palla SL, Daniels SR, Dolan LM, Kershnar AK, et al. Serum lipids and glucose control: the SEARCH for Diabetes in Youth study. Arch Pediatr Adolesc Med. 2007; 161:159-65.

52. Kamara NT, Asiimwe S. Dyslipidaemia and hypertension among adults with diabetes in rural Uganda. Trop Doct.2010; 40:41-2.
53. Subbalakshmi NK, Sathyanarayana Rao KN, Sheila R Pai. A comparative study oncardiovascular parameters and lipids in metabolic syndrome with and without diabetes mellitus.IJIRSET.2013; 11(2):1-3

54. Nasri H, Behradmanesh S, Ahmadi A, Baradaran A, Nasri P, Rafieian-Kopaei M. Association of serum lipids with level of blood pressure in type 2 diabetic patients. J Renal Inj Prev. 2014; 3(2): 4346.

55. Qiao S, Ye Q, Dou Y, Li M, Kou Y, Qian D, et al. Analysis for hypertension and related risk factors of physical examination population. Int J Clin Exp Med.2013; 6:785-93.

56. Sun NL, Wang HY, Huo Y. Blood pressure control and glucose metabolism status in hypertension specialty clinics in China. Zhonghua Nei Ke $\mathrm{Za}$ Zhi.2013; 52:654-8.

57. Basit A, Hydrie MZ, Hakeem R, Ahmedani MY, Waseem M. Glycemic control, hypertension and chronic complications in type 2 diabetic subjects attending a tertiary care center. J Ayub Med Coll Abottabad.2005; 17: 63-80.

58. Singh G, Kumar A. Impact of Chronicity on Lipid Profile of Type 2 Diabetics. Journal of Exercise Science and Physiotherapy.2013; 9(1): 46-50.

59. Verma M, Paneri S, Badi P, Raman P.G. Effect of increasing duration of diabetes mellitus type 2 on Glycated hemoglobin and insulin sensitivity. Indian J.Clini Biochemistry.2006; 21 (1):142-146.

60. Agrawal J, Bharihoke N, Kar A. Moderate correlation of fasting blood sugar with different lipid Parameters may a signal for insulin resistance in normal population. Int J Pharm Pharm Sci. 2014; 6(5): 683-685.

61. Guzder R.N, Gatting W, Mullee M.A. Prognostic value of the Framingham cardiovascular risk equation and the UKPDS risk engine for coronary heart disease in newly diagnosed type- 2 diabetes. Diabetes Med.2005; 22: 554-62.

62. Talat N, Khan A, Gulsena M, Bilal B. Dyslipidemias in Type 2 Diabetes Mellitus Patients in a Teaching Hospital of Lahore, Pakistan. Pak. J. Med. Sci.2003; 19: 283-6. 